\title{
Overexpressed circPVT1 in oral squamous cell carcinoma promotes proliferation by serving as a miRNA sponge
}

\author{
TIANPENG HE, XIN LI, DONGMEI XIE and LILI TIAN \\ Department of Stomatology, China-Japan Friendship Hospital, Beijing 100029, P.R. China
}

Received September 12, 2018; Accepted March 15, 2019

DOI: $10.3892 / \mathrm{mmr} .2019 .10615$

\begin{abstract}
Circular RNAs (circRNAs) comprise a novel class of widespread non-coding RNAs that may regulate gene expression in eukaryotes. However, the characterization and function of circRNAs remain elusive in human cancer, including oral squamous cell carcinoma (OSCC). In this study, the expression level of circPVT1 in OSCC was detected and define its functional role in initiation and progression of OSCC. It was identified that circPVT1 was upregulated in OSCC cells and specimens. Knockdown of circPVT1 suppressed cell proliferation as evidenced by Cell Counting kit-8 assay and elevated Ki-67 expression. Mechanistically, it was demonstrated that circPVT1 possessed two targeting sites of microRNA (miRNA/miR)-125b and could effectively sponge miR-125b to release its downstream mRNA targets. Subsequently, the downstream target signal transducer and activator of transcription 3 (STAT3) was verified as a direct target of miR-125b and STAT3 expression was regulated by the circPVT1/miR-125b axis. CircPVT1 functioned as competing endogenous RNA (ceRNA) to increase the STAT3 level and cell proliferation through sponging miR-125b. In conclusion, circPVT1 regulates cell proliferation and may serve as a promising therapeutic target for OSCC patients. Therefore, silencing of circPVT1 could be a future direction to develop a novel treatment strategy.
\end{abstract}

\section{Introduction}

Oral squamous cell carcinoma (OSCC) is one of the most aggressive neoplasms among head and neck malignant tumors (1). Smoking and alcohol consumption are the main environmental factors affecting the development of OSCC (2). Each year $~ 540,000$ novel cases are diagnosed and mortality

Correspondence to: Dr Lili Tian, Department of Stomatology, China-Japan Friendship Hospital, 2 Yinghua East Street, Chaoyang, Beijing 100029, P.R. China

E-mail: tianll_1974@163.com

Key words: oral squamous cell carcinoma, circularPVT1, competing endogenous RNA, signal transducer and activator of transcription 3, cell proliferation rates have not been lowered significantly in recent years. Although several advances have been obtained in its treatment, the prognosis of OSCC remains poor, with a 5-year survival ratio of nearly $50 \%$ (3). Better understanding of the genetic and molecular disorders of the disease is the key to early diagnosis, appropriate treatment and improved prognosis of patients with OSCC.

Circular RNAs (circRNAs) are another class of non-coding RNAs that are widely expressed in mammals besides microRNAs and long non-coding (lnc) RNAs (4,5). They are formed by exon skipping or back-splicing events with neither 5 ' to 3 ' polarity nor a polyadenylated tail. Nowadays, circRNAs have been extensively identified among a variety of transcriptomes. Previously, they were mostly misinterpreted as splicing errors. Only in recent years, circRNAs were rediscovered from RNA sequencing (RNA-seq) data and demonstrated to be widespread and diverse in eukaryotic cells $(6,7)$. This discovery has permanently altered perspectives toward cancer, especially in carcinogenesis and cancer progression $(8,9)$. However, the current understanding of the molecular characteristics and functional role of circRNAs in carcinogenesis remains limited.

CircPVT1, alternatively named hsa_circ_0001821, is derived from exon 3 within the oncogene PVT1 locus, which is located on chr8:128902834-128903244, a cancer susceptibility locus (10). Previously, PVT1 has been reported as an oncogene during tumor growth and metastasis (11), however, the expression and functional role of circPVT1 are not well known, including in OSCC. Previously, Chen et al (12) demonstrated that circPVT1 is a proliferative factor in gastric cancer by serving as a competing endogenous (ce)RNA. More recently, circPVT1 was identified to be upregulated in head and neck squamous carcinoma and mediated by the mutant p53/YAP/TEAD transcription-competent complex (13). Therefore, the role of circPVT1 in OSCC progression may be important.

In this study, it was hypothesized that circPVT1 is upregulated in OSCC and promotes cell proliferation by acting as a ceRNA. To test this hypothesis, circPVT1 levels were measured in OSCC cell lines. In addition, the oncogenic role of circPVT1 was also verified by performing several gain-or loss-functional assays.

\section{Materials and methods}

Clinical tissue samples. The present study included 50 patients with OSCC who underwent partial or total surgical resection at 
the Department of Otorhinolaryngology Head and Neck Surgery of the China-Japan Friendship Hospital between January 2015 and June 2017. The patients were enrolled between the ages of 28 and 79 years old and the median age at the time of diagnosis was 53 years. The male to female ratio was $37: 13$. Tissues were obtained from the tongue (21 cases), gingiva (12 cases), bucca ( 9 cases) and floor of the mouth ( 8 cases). Primary cancer tissues and adjacent non-tumor tissues $(>2 \mathrm{~cm}$ distal from cancer area to avoid the encroachment of cancer cells) were collected. No patients received radiotherapy prior to the surgical resection. The obtained tissue samples were immediately snap-frozen in liquid nitrogen upon resection and then stored at $-80^{\circ} \mathrm{C}$ until use. The present study was approved by the Research Scientific Ethics Committee of the China-Japan Friendship Hospital. All participants signed informed consent prior to using the tissues for scientific research.

Cell culture. A total of two OSCC cell lines (SCC-9 and CAL-27) were purchased from the Chinese Academy of Sciences (Shanghai, China) and normal oral epithelial keratinocytes (HOK) from ScienCell Research Laboratories (San Diego, CA, USA). The source of the OSCC cell lines was derived from tongue cancer and has been authenticated in oral cancer research (14). CAL-27 and SCC-9 cells were cultured in Dulbecco's modified Eagle's medium: Nutrient Mixture F-12 (DMEM/F12, Gibco; Thermo Fisher Scientific, Inc.) with $10 \%$ fetal bovine serum (FBS; Interpath Services Pty, Ltd.). HOK was cultured in DMEM supplemented with $10 \%$ FBS and penicillin-streptomycin $(100 \mathrm{unit} / \mathrm{ml}-100 \mu \mathrm{g} / \mathrm{ml})$. The culture condition is $37^{\circ} \mathrm{C}$ with a $5 \% \mathrm{CO}_{2}$ containing air.

Vector construction and cell transfection. The specific silencing RNAs against circPVT1 small interfering (si)-circPVT1, si-signal transducer and activator of transcription 3 (si-STAT3) or negative control and miRNA mimics (miR-125b and negative control) were purchased from Sangon Biotech Co., Ltd., (Shanghai, China). The full sequences of circPVT1 and negative control sequences were amplified and synthesized in plasmids between two frames. In total, $5 \times 10^{5}$ cells were cultured in each well of a six-well plate and were transfected with above-mentioned vectors using Lipofectamine 3000 (Invitrogen; Thermo Fisher Scientific, Inc.) according to the manufacturer's protocol. The nucleic acids were transfected at a concentration of $100 \mathrm{nM}$. Cells were harvested following $48 \mathrm{~h}$ for validation and subsequent functional analysis. The sequences of the oligonucleotides are presented in Table I.

RNA extraction and reverse transcription-quantitative polymerase chain reaction ( $R T-q P C R)$. RNA extraction from cell fraction and tissue samples was performed using a TRIzol regent (Invitrogen; Thermo Fisher Scientific, Inc.). RT and qPCR kits were used to evaluate the expression of target RNAs. The $20 \mu 1$ RT reactions were performed using a PrimeScript ${ }^{\mathbb{B}}$ RT reagent kit (Takara Bio, Inc.) and incubated for $30 \mathrm{~min}$ at $37^{\circ} \mathrm{C}, 5 \mathrm{sec}$ at $85^{\circ} \mathrm{C}$. For assaying circRNAs, mRNAs and miRNAs expression levels, the RT-qPCR analyses were performed using SYBR Premix Ex Taq ${ }^{\mathrm{TM}}$ kit (Takara) and a StepOnePlus Real-Time PCR System (Applied Biosystems; Thermo Fisher Scientific, Inc.). The values of $2^{-\Delta \Delta C q}$ relative to one of the samples was calculated to analyze relative expression levels of the genes (15). GAPDH and U6 were used to normalize the relative expression levels of circRNAs and miRNAs, respectively. The relative expression level of different genes was calculated using the $\Delta \Delta \mathrm{Cq}$ method. The primer sequences were presented in Table I.

Cell Counting kit-8 (CCK-8) assay. The proliferation of transfected cells was detected by CCK-8 (Dojindo Molecular Technologies, Inc.). A total of $\sim 2 \times 10^{3}$ OSCC cells were seeded in 96-well plates in triplicate. CCK-8 solution $(10 \mu \mathrm{l})$ was added into each well and incubated at $37^{\circ} \mathrm{C}$ for $2.5 \mathrm{~h}$. The optical density value at $450 \mathrm{~nm}$ was measured using the SpectraMax M5 microplate reader (Molecular Devices, LLC).

Cell cycle assay. A total of $>1 \times 10^{4}$ transfected cells were harvested and fixed with $75 \%$ ethanol at $4^{\circ} \mathrm{C}$ for $24 \mathrm{~h}$. The fixed cells were washed with cold PBS twice and mixed with propidium iodide (PI; cat. no. 550825; BD Biosciences) for $20 \mathrm{~min}$ at room temperature. Cell cycle analysis was performed by flow cytometry analysis using a BD FACSCalibur (BD Biosciences) flow cytometer and the data were analyzed using the ModFit LT software (version 4.1; Verity Software House, Inc.).

Bioinformatics analysis. The putative binding sites of miR-125b in the circPVT1 sequence were predicted using miRBase (http://www.mirbase.org/) and TargetScan (http://www.targetscan.org).

Nucleocytoplasmic separation. The PARIS ${ }^{\mathrm{TM}}$ kit (Ambion; Thermo Fisher Scientific, Inc.) was used for the nucleic-cytoplasmic separation experiment. Briefly, $5 \times 10^{6}$ cells were resuspended in $0.6 \mathrm{ml}$ resuspension buffer for $15 \mathrm{~min}$ 's incubation at room temperature, followed by homogenization. The pellet consisted the nuclear fraction following a $1 \times 10^{5} \times \mathrm{g}$ centrifugation at $4^{\circ} \mathrm{C}$ for $10 \mathrm{~min}$. CircPVT1 expression was determined by qPCR with GAPDH as cytoplasmic control and $\mathrm{U} 1$ as nuclear control.

Fluorescence in situ hybridization (FISH) analysis. Cy3-labeled circPVT1 probes were obtained from Guangzhou RiboBio Co., Ltd. Hybridizations were carried out using a FISH kit (Guangzhou RiboBio Co., Ltd.) according to the manufacturer's protocol. All fluorescence images were captured using Nikon A1Si Laser Scanning Confocal Microscope (Nikon Corporation).

Immunofluorescence. Cell were permeabilized with 0.3\% Triton X-100 (Beyotime Institute of Biotechnology) at room temperature for $15 \mathrm{~min}$ after being fixed with $4 \%$ paraformaldehyde for $30 \mathrm{~min}$ at room temperature. Cells were blocked using 5\% goat serum (Beyotime Institute of Biotechnology) for $30 \mathrm{~min}$ at room temperature, and then were incubated with an anti-Ki67 antibody (1:100; cat. no. ab15580; Abcam) overnight at $4^{\circ} \mathrm{C}$. The slides were then incubated with an anti-rabbit Alexa Fluor 488-labeled secondary antibody (1:500; cat. no. 016-540-084; Jackson ImmunoResearch Laboratories, Inc.) for $1 \mathrm{~h}$ at room temperature. DAPI was used for nuclear counterstaining at $4^{\circ} \mathrm{C}$ for $10 \mathrm{~min}$. The samples were observed under a fluorescence microscope (IX73; Olympus Corporation; magnification, $\mathrm{x} 20$ ). 
Table I. Information of the RT-qPCR primer sequences and oligonucleotides sequences.

RT-qPCR primer name

CircPVT1 Forward

CircPVT1 Reverse

GAPDH Forward

GAPDH Reverse

U6 Forward

U6 Reverse

U1 Forward

U1 Reverse

Oligonucleotide name

si-PVT1 (1)

si-PVT1 (2)

si-STAT3

si-NC

miR-125b

miR-NC

Oligos for plasmid construction

circPVT1-exp-vector-F

circPVT1-exp-vector-R

LUC-UTRcricPVT1-F

LUC-UTRcricPVT1-R
Sequence (5'-3')

ATCGGTGCCTCAGCGTTCGG
CTGTCCTCGCCGTCACACCG
GCACCGTCAAGGCTGAGAAC
ATGGTGGTGAAGACGCCAGT
CTCGCTTCGGCAGCACA
AACGCTTCACGAATTTGCGT
GGGAGATACCATGATCACGAA
CCACAAATTATGCAGTCGAGTT

UGGGCUUGAGGCCUGAUCU

GCUUGAGGCCUGAUCUUUU

GCAGCAGCTGAACAACATG

UUCUCCGAACGUGUCACGUTT

UCCCUGAGACCCUAACUUGUGA

CGAAUUAACGGGCCUAUCCGAU

gcagaattcAGAGGCAGCTCTCACCTGAA gcaggtaccTCAGAAAATGGTGACAGGTA gcagaattcGCCTGATCTTTTGGCCAGAA gcaggatcCAGCCTCACCTCAAGCCCAG

RT-qPCR, reverse transcription-quantitative polymerase chain reaction; si, small interfering; NC, negative control; STAT3, signal transducer and activator of transcription; UTR, untranslated region; F, forward; R, reverse.

RNA immunoprecipitation (RIP). The RIP assay was performed using the Magna RIP ${ }^{\mathrm{TM}}$ RNA-Binding Protein Immunoprecipitation kit (EMD Millipore). Briefly, the cells were harvested and resuspended in the NP-40 lysis buffer (1 mM; Beyotime Institute of Biotechnology) for $5 \mathrm{~min}$ at room temperature following $48 \mathrm{~h}$ of transfection. A total of $10 \mu \mathrm{l}$ of the cell lysates were sampled for input and the remaining lysates were incubated with anti-STAT3 antibody (1:100; cat. no. ab119352; Abcam) or negative control immunoglobulin $\mathrm{G}$ (1:100; cat. no. 12-370; EMD Millipore) at $4^{\circ} \mathrm{C}$ overnight. Next, the beads were washed with wash buffer and the RNAs captured by beads were extracted using the RNeasy Mini kit (Qiagen, Inc.). Finally, RT-qPCR was performed to detect the enrichment and analyze. The magnetic bead bound complexes were immobilized with a magnet and unbound materials were washed off. Then, RNAs were extracted and analyzed by qPCR.

Luciferase reporter assay. OSCC cells were seeded in 24-well plates at a density of $5 \times 10^{3}$ cells per well and maintained for $24 \mathrm{~h}$. A total of $250 \mathrm{ng}$ Firefly Luciferase (FL) reporter, $500 \mathrm{ng}$ Renilla luciferase reporter vectors and miR-125b mimics/NC mimics were co-transfected into the cells, and the cells were harvested in $48 \mathrm{~h}$. The luciferase activity was measured with a dual luciferase reporter assay detection kit (Promega Corporation) on an Omega device (Omega Bio-Tek, Inc.). The Firefly luciferase activity was normalized to Renilla luciferase activity.

Western blotting and antibodies. The total proteins were extracted using RIPA buffer (Sigma-Aldrich; Merck KGaA), and their concentrations were detected using Total Protein Extraction kit (Beijing Solarbio Science \& Technology Co., Ltd.). Cell lysates ( $25 \mu \mathrm{g}$ protein in each well) were separated by $10 \%$ SDS-PAGE and transferred to nitrocellulose membranes (GE Healthcare). Then, $5 \%$ concentration non-fat milk in TBST buffer (containing $0.1 \%$ Tween-20) was used for the blockage of membranes containing protein for $2 \mathrm{~h}$ at room temperature. The antibodies used included primary antibodies anti-STAT3 (1:1,000; Abcam; cat. no. ab65222), anti-GAPDH (1:1,000; cat. no. 5174; Cell Signaling Technology, Inc.), which were incubated with membranes at $4^{\circ} \mathrm{C}$ overnight; horseradish peroxidase-conjugated secondary goat anti-mouse (1:5,000; cat. no. SA00001-1) or goat anti-rabbit $(1: 5,000$; cat. no. SA00001-2; both ProteinTech Group, Inc.) antibodies ( $2 \mathrm{~h}$ at room temperature). The relative grey values of immunoreactive bands were calculated based on GAPDH.

Statistical analysis. All experiments were performed in triplicate. Data are presented as the mean \pm standard error of the mean and analyzed using GraphPad Prism 6.0 (GraphPad Software, Inc.). Parametric pared t-test was performed to compare the difference between OSCC tissues and paired normal tissues. A Chi-squared test was used to analyze the correlation between clinicopathological features of OSCC patients and circPVT1 expression. One-way ANOVA was performed to evaluate difference among multiple groups. Fisher's Least Significant Difference test was used as post-hoc test. Receiver operation characteristic (ROC) analysis was 
A

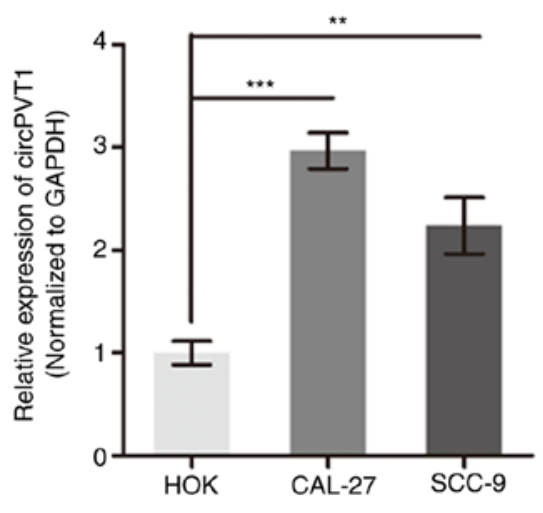

B

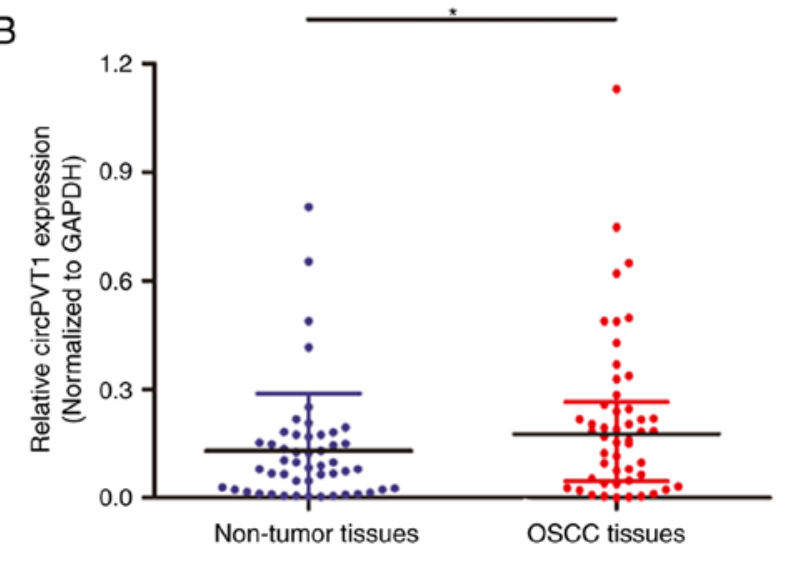

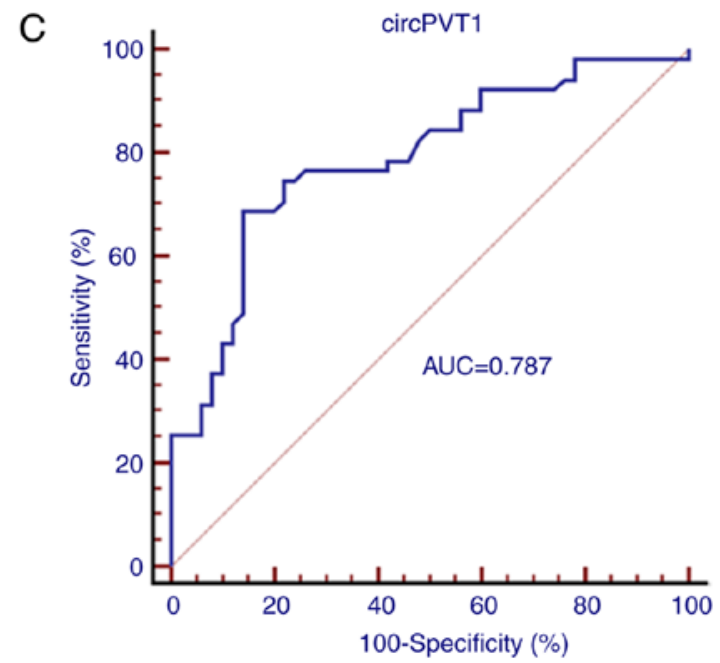

Figure 1. Knockdown of circPVT1 regulates cell proliferation and cell cycle phase in OSCC. (A) RT-qPCR analysis of circPVT1 expression in OSCC cell lines and human normal oral epithelial keratinocytes, ${ }^{* *} \mathrm{P}<0.01$ and ${ }^{* * *} \mathrm{P}<0.001$ vs. the HOK cells. (B) RT-qPCR analysis of circPVT1 in 50 primary OSCC tissues and paired non-tumor tissues, ${ }^{\prime} \mathrm{P}<0.05$. (C) Receiver operation characteristic curve was established using the expression of circPVT1 in 50 primary OSCC tissues and paired non-tumor tissues. RT-qPCR, reverse transcription-quantitative polymerase chain reaction; OSCC, oral squamous cell carcinoma; circ, circular; AUC, area under the curve.

performed using MedCalc version 11.4 (MedCalc Software bvba) to evaluate the diagnostic performance of circPVT1. $\mathrm{P}<0.05$ was considered to indicate a statistically significant difference.

\section{Results}

CircPTVI is upregulated in OSCC. To determine the potential role of circPVT1 in OSCC, its expression level was measured in OSCC by RT-qPCR. As presented in Fig. 1A, circPVT1 was significantly upregulated in two OSCC cell lines, CAL-27 and SCC-9, when compared to the normal oral epithelial keratinocytes, HOK cells $(\mathrm{P}<0.01)$. In addition, circPVT1 level was also significantly increased in 50 OSCC tissues compared with precancerous tissues $(\mathrm{P}<0.05$; Fig. $1 \mathrm{~B})$. Following validation of circPVT1 upregulation in OSCC patients, the diagnostic role of circPVT1 was investigated by establishing ROC curves using the 50 paired OSCC tissues (Fig. 1C). The area under the curve of the ROC curve was 0.787 and the detective sensitivity, and specificity were 68.6 and $86.0 \%$ respectively. Analysis of association between circPVT1 expression and clinical pathological factors of revealed that circPVT1 expression was positively correlated with tumor size and tumor node and metastasis, but not with other factors, including sex, age and histological grade (Table II). This indicates a potential prognostic significance of circPVT1 for OSCC patients.

Knockdown of circPVT1 inhibits cell proliferation in OSCC. For the upregulated expression levels of circPVT1 in OSCC cells, the present study hypothesized that circPVT1 may serve an oncogenic role in biological processes of OSCC. To verify the hypothesis, specific siRNAs targeting circPVT1 were constructed (Fig. 2A) to silence its expression in cell lines CAL-27 and SCC-9 cells and perform several experiments. As si-circPVT1 (1) demonstrated a better silencing efficiency, it was selected for the following experimental assays (Fig. 2B). Cell proliferation was determined by performing a CCK- 8 assay and the results demonstrated that silencing of circPVT1 suppressed OSCC cell growth in contrast to the negative controls (Fig. 2C). Immunofluorescence analysis using Ki-67 antibody demonstrated that silencing circPVT1 decreased Ki-67 expression level in (Fig. 2D), which is a well-known proliferative protein marker. Meanwhile, the cell cycle assay indicated that knockdown of circPVT1 induced cell cycle arrest at the $\mathrm{G}_{2} / \mathrm{M}$ phase in CAL-27 and SCC-9 cells compared with controls. Therefore, the results of the present 
Table II. Clinical characteristics of 50 patients and the expression of circPVT1 in primary oral squamous cell carcinoma tissues.

\begin{tabular}{lccc}
\hline Characteristics & Case & $\begin{array}{c}\text { circPVT1 } \\
\text { median (range) }\end{array}$ & P-value \\
\hline $\begin{array}{l}\text { Age (years) } \\
\quad 55\end{array}$ & 25 & $0.15(0.02-0.67)$ & 0.776 \\
$\quad \geq 55$ & 25 & $0.17(0.06-0.82)$ & \\
Sex & & & 0.275 \\
$\quad$ Male & 34 & $0.18(0.02-0.82)$ & \\
Female & 16 & $0.11(0.04-0.67)$ & \\
Tumor size & & & 0.021 \\
$\quad<5$ cm & 33 & $0.10(0.04-0.74)$ & \\
$\geq 5$ cm & 17 & $0.28(0.01-0.82)$ & \\
Histological grade & & & 0.106 \\
$\quad$ Well & 11 & $0.13(0.01-0.64)$ & \\
Moderate & 27 & $0.17(0.05-0.61)$ & \\
Poor & 12 & $0.21(0.08-0.82)$ & \\
TNM stage & & & 0.002 \\
I-II & 23 & $0.09(0.01-0.56)$ & \\
III-IV & 27 & $0.26(0.06-0.82)$ & \\
& &
\end{tabular}

circ, circular; TNM, tumor, node and metastasis.

study indicate circPVT1 promoted proliferation of OSCC cells.

CircPVT1 serves as a ceRNA via sponging miR-125b in $O S C C$. To test whether circPVT1 functions as a ceRNA in OSCC, the subcellular location of circPVT1 in OSCC cells was first determined. A nucleocytoplasmic separation assay demonstrated that circPVT1 may be distributed mainly in the cell cytoplasm (Fig. 3A). The FISH assay with specific probe verified circPVT1 was located in the cytoplasm (Fig. 3B). Subsequently, whether circPVT1 influenced the biofunctions of OSCC cells was investigated through sponging of miRNAs. As expected, miR-125b was verified as a potential sponged miRNA with two targeting sites within circPVT1 according to miRBase and TargetScan (Fig. 3C). Using two OSCC cell lines, it was identified that knockdown of circPVT1 significantly promoted miR-125b expression ( $\mathrm{P}<0.01$; Fig. 3D). Then, a luciferase reporter assay was performed by generating miR-125b mimics (Fig. 3E). As expected, the results demonstrated that miR-125b mimics significantly silenced the luciferase activity of circPVT1 (Fig. 3F) but exhibited no effect on mutant controls. Taken together, the sponging of miR-125b by circPVT1 in OSCC cells was verified.

CircPVT1 affects the STAT3 expression level through sponging $m i R-125 b$. Previously, it was reported that STAT3 was directly targeted by miR-125b $(16,17)$. Furthermore, STAT3 is known to serve a key role in a number of cellular processes including cell growth (18). Therefore, whether STAT3 is essential for the OSCC proliferation change mediated by the circPVT1/miR-125b pathway was investigated. Using bioinformatics analysis, STAT3 was predicted to be targeted by miR-125b (Fig. 4A). miR-125b mimics suppressed STAT3 expression level in OSCC cells (Fig. 4B). Meanwhile, knockdown of circPVT1 decreased STAT3 levels (Fig. 4C). RIP experiment revealed that miR-125b was pulled down by STAT3 antibody. Furthermore, the enrichment between miR-125b and STAT3 was increased when circPVT1 was silenced in CAL-27 cells (Fig. 4D). These indicate that circPVT1 released STAT3 by binding to miR-125b in OSCC.

CircPVT1 promotes cell proliferation via sponging $m i R-125 b$ and releasing STAT3. Full-length cDNA of circPVT1 from CAL-27 cells was generated and loaded into the plasmid vectors (Fig. 5A). The upregulation of circPVT1 in OSCC cells was also confirmed following transfection of the vector by qPCR (Fig. 5B). In addition, STAT3 was silenced by transfection of specific siRNA (Fig. 5C). Next, the CCK-8 assay demonstrated that circPVT1 promoted the cell growth of OSCC cells, however, the enhanced cell growth was partially reversed by knockdown of STAT3 or overexpression of miR-125b (Fig. 5D). Furthermore, immunofluorescence demonstrated an elevated expression of $\mathrm{Ki}-67$ by overexpression of circPVT1 and this elevation was reversed by co-expression of miR-125b or si-STAT3 (Fig. 5E). To conclude, circPVT1 may promote cell growth by sponging miR-125b and therefore increasing STAT3 expression.

\section{Discussion}

Numerous studies have been performed to get a thorough understanding of biological characteristics and functional roles of noncoding RNAs in cancer progression; however, their specific roles are still largely unknown in cancer, including OSCC $(19,20)$. More recently, circRNA have been increasingly recognized as oncogenes or tumor-suppressor genes in different types of cancers, including gastric cancer (21), breast cancer (22) and colorectal cancer (23). However, the role of circRNAs in OSCC is largely unknown. In this study, the expression and function of circPVT1 in OSCC was investigated. The results of the present study demonstrated that increased expression of circPVT1 in OSCC could enhance cell proliferation via sponging miR-125b and upregulating the STAT3 expression level.

During the past years, miRNAs and lncRNAs have been investigated to be used as potential diagnostic predictors or therapeutic targets $(24,25)$. However, the outcome is not satisfactory and the translational studies have grown stagnant. In the present study, the novel findings of circRNAs open up a new area. As non-classical products of transcription, circRNAs are thought to be synthesized co-transcriptionally in the nucleus and are modified in the cytoplasm (26). CircRNAs can selectively bind to target miRNAs in a sequence specific manner to form the circRNA-miRNA complexes that block the functions of miRNAs (27). Unlike known unidimensional lncRNAs, circRNAs in general are produced from pre-mRNAs back-spicing events, which covalently linked the $3^{\prime}$ and $5^{\prime}$ ends of exons or introns together and formed single-stranded continuous loop structures with neither a $5^{\prime}$ cap nor a $3^{\prime}$ polyadenylated tail. Compared to linear RNAs, circRNAs are much more resistant to RNase $\mathrm{R}$ and more stable probably due 
A

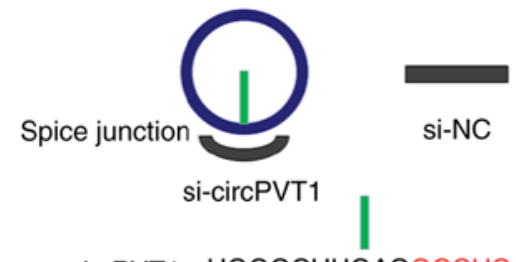

circPVT1 ...UGGGCUUGAGGCCUGAUCUUUUG... si-circPVT1 (1) CUUGAGGCCUGAUCUUUUG si-circPVT1 (2) UGGGCUUGAGGCCUGAUCU

$\mathrm{C}$ CAL-27
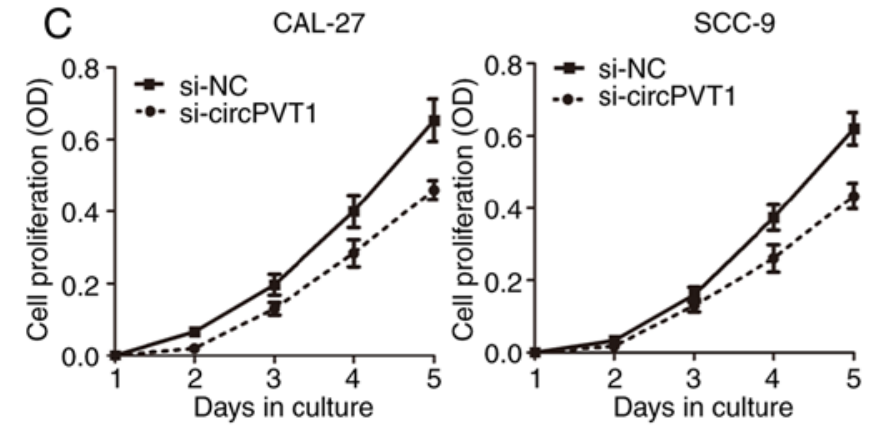

B

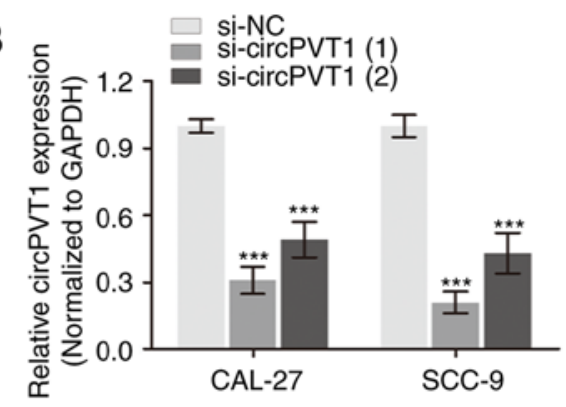

$\mathrm{D}$

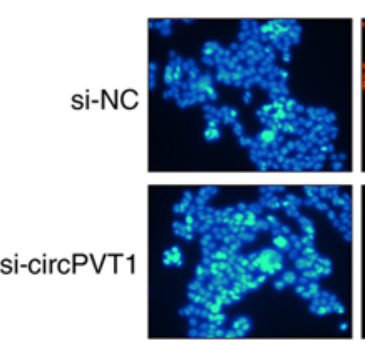

Merge
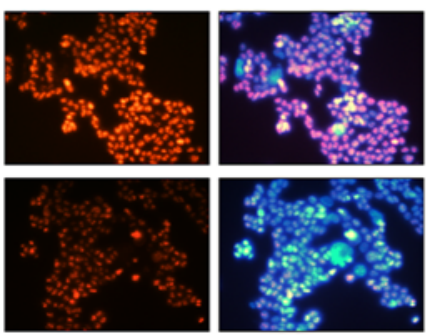
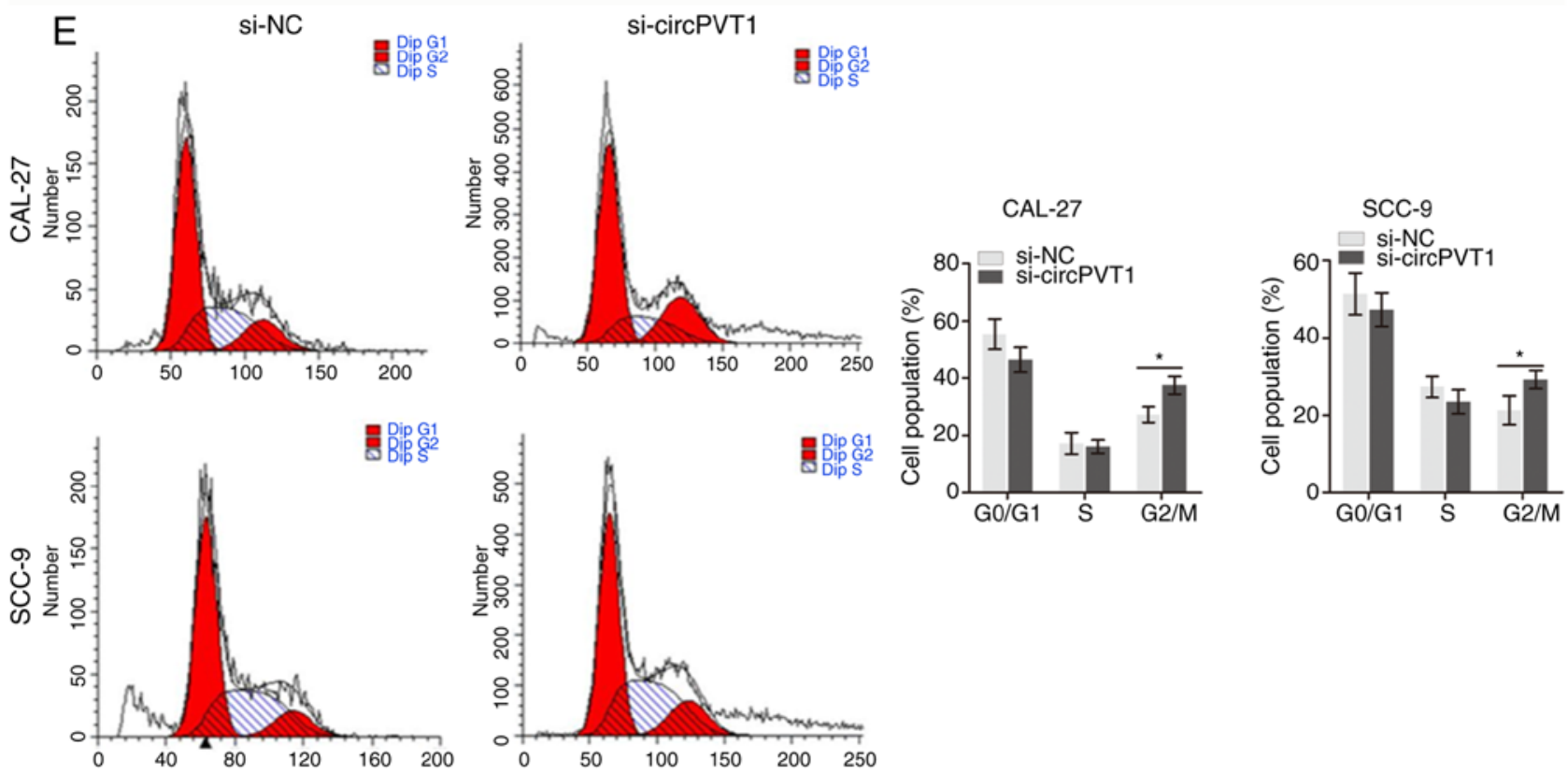

Figure 2. Knockdown of circPVT1 suppresses cell proliferation of OSCC. (A) The established vector used for silencing circPVT1 and negative controls vector are presented (B). The expression of circPVT1 in cells transfected with silencing vector or controls were measured via quantitative polymerase chain reaction, ${ }^{* * *} \mathrm{P}<0.001$ vs. the si-NC group. (C) Cell Counting kit- 8 analysis demonstrated the suppressive effect of silencing circPVT1 on cell proliferation. (D) Immunofluorescence assay demonstrated that knockdown of circPVT1 suppressed Ki-67 expression in CAL-27 cells. Magnification, x20. (E) The influence induced by knockdown of circPVT1 on cell cycle mode are presented, ${ }^{*} \mathrm{P}<0.05$. OSCC, oral squamous cell carcinoma; circ, circular; AUC, area under the curve; si, small interfering; NC, negative control.

to the absence of $5^{\prime}$ and $3^{\prime}$ ends $(28,29)$. Importantly, it was identified that one circRNA named circPVT1 was upregulated in OSCC tissues and cells, which indicates that circPVT1 has the potential to serve as useful biological marker and may be critical in OSCC progression.

Several studies have demonstrated that the expression profiles of circRNAs are aberrant in diverse cancer types and certain ones serve very important roles in oncogenesis and cancer development, including as circPVT1 $(13,30,31)$. The specific role of circPVT1 in OSCC is still unknown. In this study, it was identified that circPVT1 was downregulated in
OSCC compared to noncancerous tissues and predominantly localized in the cytoplasm. Importantly, circPVT1 could directly sponge miR-125b to release STAT3 and subsequently promotes proliferation of OSCC by upregulating STAT3 expression. Therefore, the aforementioned studies suggest that circPVT1 serves quite important roles in neoplasia and cancer development. In addition, it was reported that $\mathrm{G}_{2} / \mathrm{M}$ arrest was involved in cellular dormancy. Therefore, the role of circPVT1 in dormancy still needs to be further investigated.

It was demonstrated that circPVT1 was enriched in the cytoplasm section and revealed that it acts as a miRNA sponge 

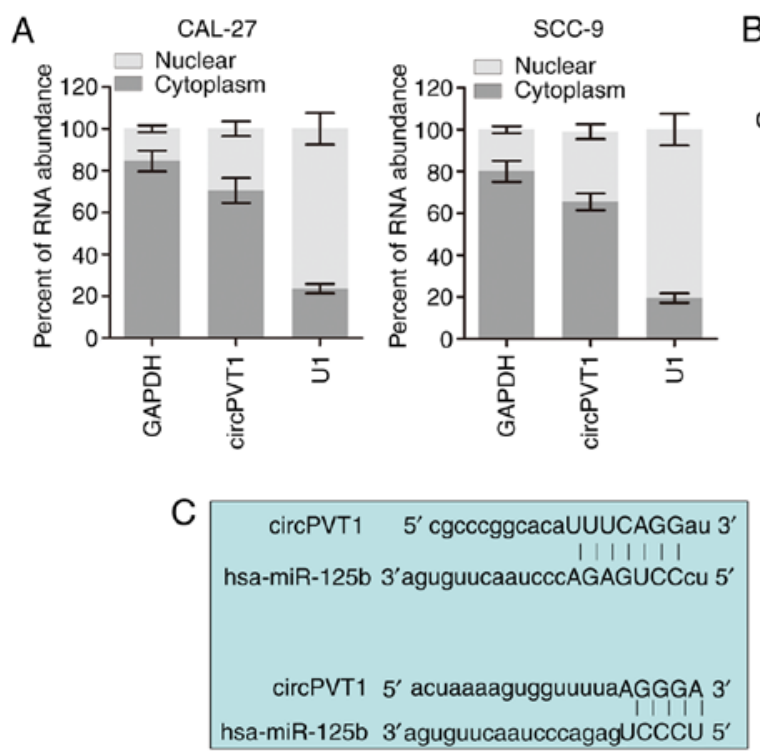

B
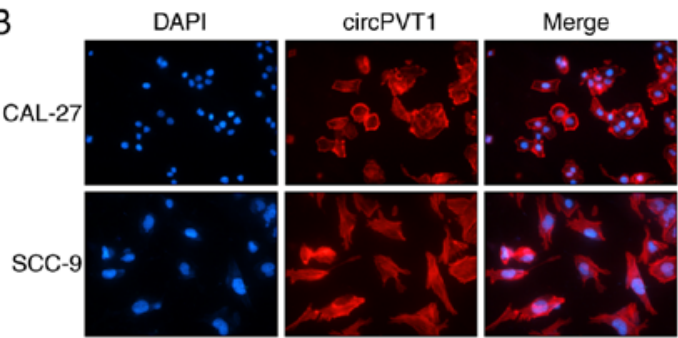

D $\stackrel{9}{3} \quad 3 \quad$ si-NC

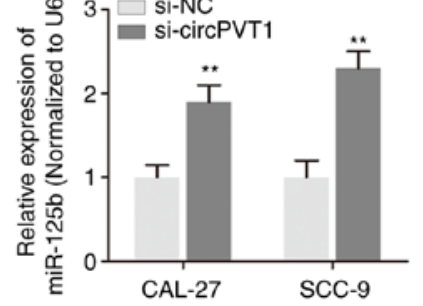

E

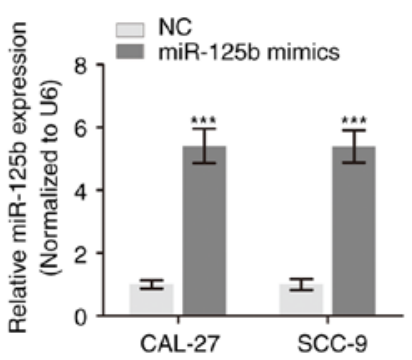

$\mathrm{F}$

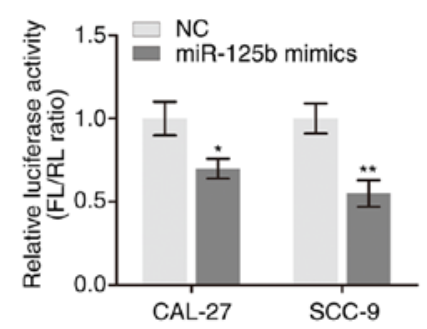

PGL3-circPVT1 (Mu)

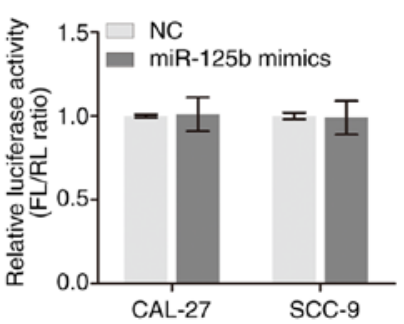

Figure 3. circPVT1 serve as a competing endogenous RNA of miR-125b. (A) The expression level of circPVT1 in nuclear and cytoplasm of OSCC cells were verified via reverse transcription-qPCR. (B) The expression density of circPVT1 in OSCC cells was determined using fluorescence in situ hybridization assay. Magnification, $x 40$. (C) The putative sequences of miR-125b and circPVT1 with two binding sites. (D) miR-125b expression was increased in OSCC cells following knockdown of circPVT1. ${ }^{* *} \mathrm{P}<0.01$ vs. the si-NC group. (E) qPCR validation of overexpression of miR-125b by transfection of miR-125b mimics. ${ }^{* * *} \mathrm{P}<0.001$ vs. the NC group. (F) Firefly luciferase activity normalized to Renilla luciferase activity (FL/RL) in OSCC cells co-transfected with luciferase reporters with Wt or $\mathrm{Mu}$ transcripts of circPVT1. ${ }^{*} \mathrm{P}<0.05$ and ${ }^{* *} \mathrm{P}<0.01$ vs. the $\mathrm{NC}$ group. qPCR, quantitative polymerase chain reaction; $\mathrm{NC}$, negative control; OSCC, oral squamous cell carcinoma; circ, circular; miR, microRNA; Wt, wild type; Mu, mutant; si, small interfering; NC, negative control; miR, microRNA; FL/RL, firefly luciferase/Renilla luciferase.

A

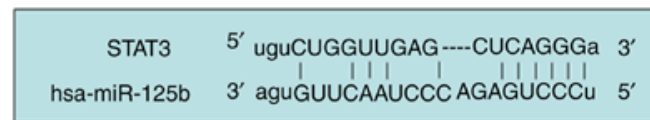

C

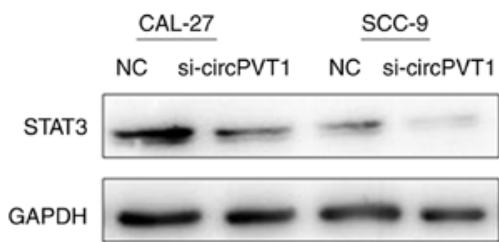

B
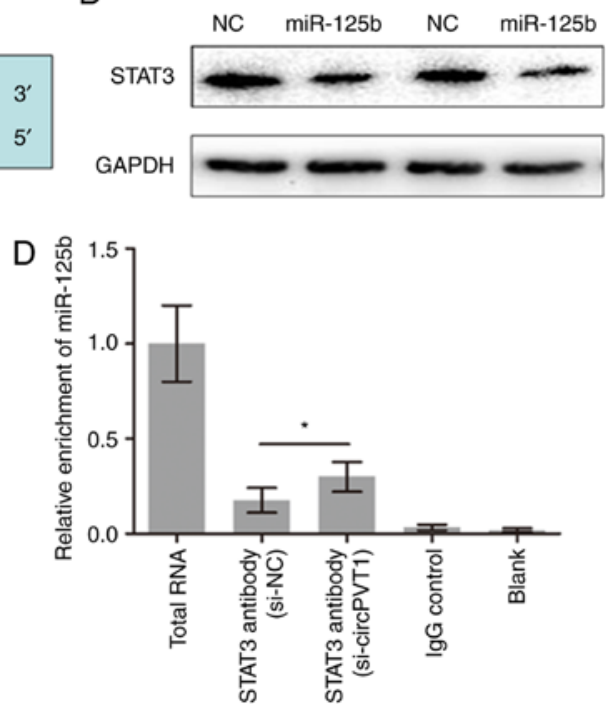

Figure 4. STAT3 mRNA is verified as a downstream target of miR-125b in OSCC. (A) The putative sequences of miR-125b and circPVT1 with one binding site. (B) Western blot analysis of STAT3 protein level in OSCC cells overexpressed miR-125b. (C) Western blot analysis of STAT3 protein level in OSCC cells silencing of circPVT1. (D) RNA immunoprecipitation was performed in cells downregulated circPVT1 using a STAT3 antibody, ${ }^{\circ}<0.05$. STAT3, signal transducer and activator of transcription; OSCC, oral squamous cell carcinoma; miR, microRNA; circ, circular; NC, negative control; si, small interfering; miR, microRNA; IgG, immunoglobulin G. 
A

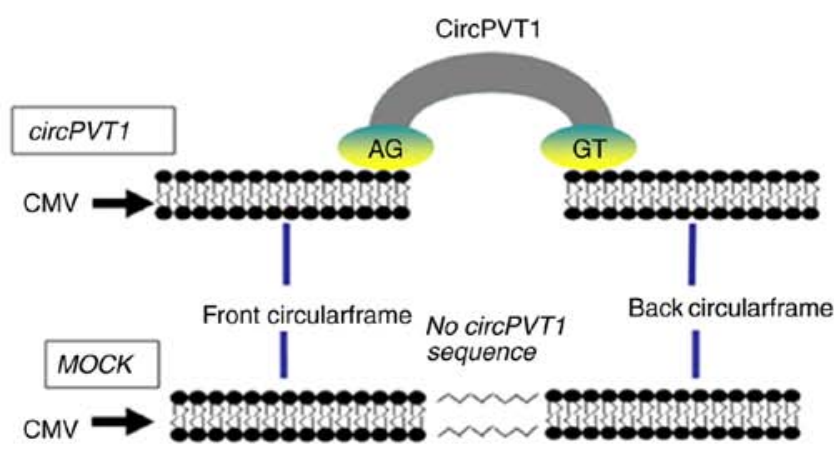

B

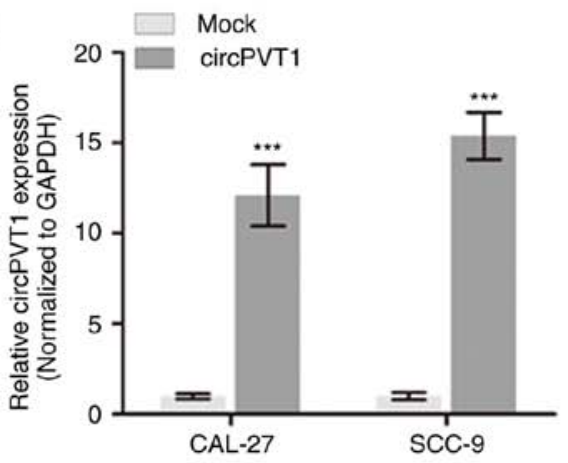

C

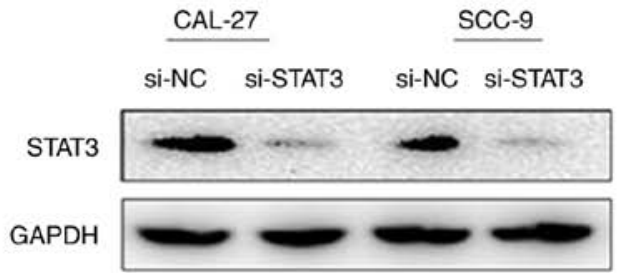

D

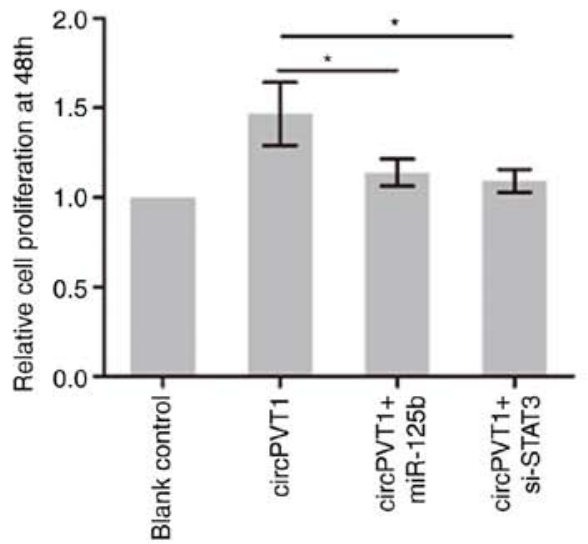

E
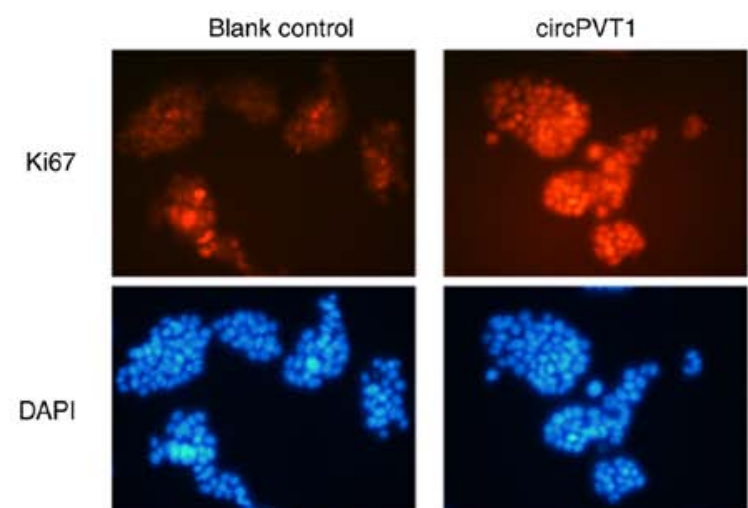

circPVT1+miR-125b

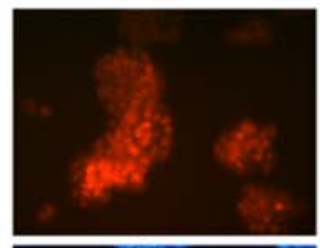

circPVT1+Si-STAT3
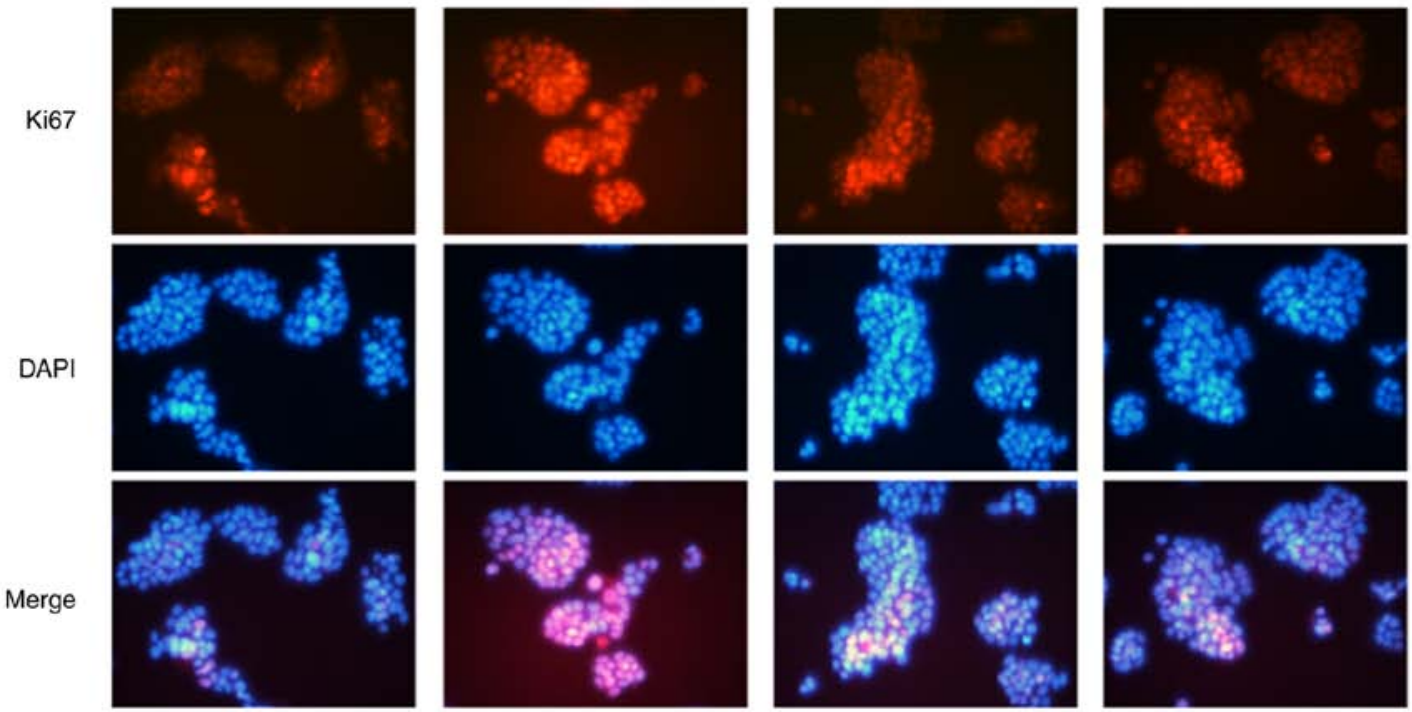

Figure 5. circPVT1 regulates cell proliferation via sponging miR-125b and releasing STAT3. (A) The established vector used for silencing circPVT1 and negative controls vector are presented. (B) Quantitative polymerase chain reaction demonstrated an upregulation of circPVT1 in cells transfected with circPVT1 overexpressing vector. ${ }^{* * *} \mathrm{P}<0.001$ vs. the Mock group. (C) Western blotting validation of decreased STAT3 protein level following transfection of STAT3 silencing vector. (D) Cell proliferation was determined following transfection for $48 \mathrm{~h}$ by Cell Counting kit- 8 assay. ${ }^{*} \mathrm{P}<0.05$. (E) Immunofluorescence analysis of Ki-67 expression level in different treatment groups. Magnification, x20. STAT3, signal transducer and activator of transcription; OSCC, oral squamous cell carcinoma; miR, microRNA; circ, circular; NC, negative control; si, small interfering; CMV, cytomegalovirus.

in OSCC to exert the regulatory function. An increasing amount of evidence suggests that miRNAs are abnormally expressed in diverse human cancer and have important roles in the tumorigenesis, progression and metastasis of these cancers (32). The first well-known circRNA serving as miRNA sponge is ciRS-7. Expression of ciRS-7 efficiently tethers miR-7, resulting in reduced miR-7 activity and increased levels of miR-7-targeted transcripts (33). After validating the direct interaction between circPVT1 and miR-125b, STAT3 mRNA was verified as a downstream target of miR-125b. STAT3 is a central regulator of numerous cellular processes, particularly cell proliferation $(34,35)$. The oncogenic property of STAT3 has been observed in multiple types of cancer due to its effect on promoting proliferation progression. However, positive and negative associations between STAT3 activation and patient survival or tumor progression have been reported (36-38). 
Therefore, the role of STAT3 in tumorigenesis seems complex and requires a careful and repeated validation. The current study suggested that STAT3 promoted cell growth and was affected by the circPVT1/miR-125b axis. Furthermore, STAT3 and miR-125b are essential for the circPVT1-induced cell proliferation, indicating the oncogenic role of STAT3.

The present study has limitations. First, the investigation of the prognostic significance of circPVT1 expression in OSCC patient survival is needed. A follow-up study will be conducted with the auhors' subsequent research work. Second, although the in vitro experimental study was repeated using two OSCC cell lines, the functional role of circPVT1 in OSCC needs a further validation with other OSCC cell lines. In the future, the functional role of circPVT1 in OSCC cell proliferation will be comprehensively validated.

In conclusion, the present study's findings revealed that overexpression of circPVT1 promoted cell proliferation via serving as a sponge for miR-125b. Therefore, circPVT1 may be a promising predictive biomarker and therapeutic target for OSCC patients.

\section{Acknowledgements}

Not applicable.

\section{Funding}

The present study was funded by The National Natural Science Foundation of China (grant no. 86722321).

\section{Availability of data and materials}

The datasets used and/or analyzed during the current study are available from the corresponding author on reasonable request.

\section{Authors' contributions}

TH and LT designed the study and performed the experiments; XL and DX performed the statistical work and wrote the manuscript; TH constructed the figures and tables and LT approved the data and the final manuscript.

\section{Ethics approval and consent to participate}

The study protocol was approved by the Clinical Research Ethics Committee of China-Japan Friendship Hospital.

\section{Patient consent for publication}

Not applicable.

\section{Competing interests}

The authors declare that they have no competing interests.

\section{References}

1. Su SC, Lin CW, Liu YF, Fan WL, Chen MK, Yu CP, Yang WE Su CW, Chuang CY, Li WH, et al: Exome sequencing of oral squamous cell carcinoma reveals molecular subgroups and novel therapeutic opportunities. Theranostics 7: 1088-1099, 2017.
2. Yan L, Chen F, Liu F, Qiu Y, Wang J, Wu J, Bao X, Hu Z, Peng X, Lin X, et al: Differences in modifiable factors of oral squamous cell carcinoma in the upper and lower of oral fissure. Oncotarget 8: 75094-75101, 2017.

3. Glazer CA, Chang SS, Ha PK and Califano JA: Applying the molecular biology and epigenetics of head and neck cancer in everyday clinical practice. Oral Oncol 45: 440-446, 2009.

4. Chen LL and Yang L: Regulation of circRNA biogenesis. RNA Biol 12: 381-388, 2015.

5. Hsiao KY, Sun HS and Tsai SJ: Circular RNA-New member of noncoding RNA with novel functions. Exp Biol Med (Maywood) 242: 1136-1141, 2017.

6. Lasda E and Parker R: Circular RNAs: Diversity of form and function. RNA 20: 1829-1842, 2014.

7. Holdt LM, Kohlmaier A and Teupser D: Molecular roles and function of circular RNAs in eukaryotic cells. Cell Mol Life Sci 75: 1071-1098, 2018

8. Gao D, Zhang X, Liu B, Meng D, Fang K, Guo Z and Li L: Screening circular RNA related to chemotherapeutic resistance in breast cancer. Epigenomics 9: 1175-1188, 2017.

9. Kristensen LS, Hansen TB, Veno MT and Kjems J: Circular RNAs in cancer: Opportunities and challenges in the field. Oncogene 37: 555-565, 2018.

10. Meyer KB, Maia AT, O'Reilly M, Ghoussaini M, Prathalingam R, Porter-Gill P, Ambs S, Prokunina-Olsson L, Carroll J and Ponder BA: A functional variant at a prostate cancer predisposition locus at $8 \mathrm{q} 24$ is associated with PVT1 expression. PLoS Genet 7: e1002165, 2011.

11. Lu D, Luo P, Wang Q, Ye Y and Wang B: lncRNA PVT1 in cancer: A review and meta-analysis. Clin Chim Acta 474: 1-7, 2017.

12. Chen J, Li Y, Zheng Q, Bao C, He J, Chen B, Lyu D, Zheng B, $\mathrm{Xu}$ Y, Long Z, et al: Circular RNA profile identifies circPVT1 as a proliferative factor and prognostic marker in gastric cancer. Cancer Lett 388: 208-219, 2017.

13. Verduci L, Ferraiuolo M, Sacconi A, Ganci F, Vitale J, Colombo T, Paci P, Strano S, Macino G, Rajewsky N and Blandino G: The oncogenic role of circPVT1 in head and neck squamous cell carcinoma is mediated through the mutant p53/YAP/TEAD transcription-competent complex. Genome Biol 18: 237, 2017.

14. Zhao M, Sano D, Pickering CR, Jasser SA, Henderson YC, Clayman GL, Sturgis EM, Ow TJ, Lotan R, Carey TE, et al: Assembly and initial characterization of a panel of 85 genomically validated cell lines from diverse head and neck tumor sites. Clin Cancer Res 17: 7248-7264, 2011.

15. Livak KJ and Schmittgen TD: Analysis of relative gene expression data using real-time quantitative PCR and the 2(-Delta Delta C(T)) method. Methods 25: 402-408, 2001.

16. Dai CY, Tsai YS, Chou WW, Liu T, Huang CF, Wang SC, Tsai PC, Yeh ML, Hsieh MY, Huang CI, et al: The IL-6/STAT3 pathway upregulates microRNA-125b expression in hepatitis $\mathrm{C}$ virus infection. Oncotarget 9: 11291-11302, 2018.

17. Gorczynski RM, Alexander C, Brandenburg K, Chen Z, Heini A, Neumann D, Mach JP, Rietschel ET, Terskikh A, Ulmer AJ, et al: Corrigendum to 'An altered REDOX environment, assisted by over-expression of fetal hemoglobins, protects from inflammatory colitis and reduces inflammatory cytokine expression' [Int. Immunopharmacol. 50 (2017) 69-76]. Int Immunopharmacol 59: 414, 2018.

18. MacPherson S, Horkoff M, Gravel C, Hoffmann T, Zuber J and Lum JJ: STAT3 regulation of citrate synthase is essential during the initiation of lymphocyte cell growth. Cell Rep 19: 910-918, 2017.

19. Momen-Heravi F and Bala S: Emerging role of non-coding RNA in oral cancer. Cell Signal 42: 134-143, 2018.

20. Li P, Zhang X, Wang H, Wang L, Liu T, Du L, Yang Y and Wang C: MALAT1 is associated with poor response to oxaliplatin-based chemotherapy in colorectal cancer patients and promotes chemoresistance through EZH2. Mol Cancer Ther 16: 739-751, 2017.

21. Yang J, Meng X, Pan J, Jiang N, Zhou C, Wu Z and Gong Z: CRISPR/Cas9-mediated noncoding RNA editing in human cancers. RNA Biology 15: 35-43, 2018.

22. Li P, Chen S, Chen H, Mo X, Li T, Shao Y, Xiao B and Guo J: Using circular RNA as a novel type of biomarker in the screening of gastric cancer. Clin Chim Acta 444: 132-136, 2015.

23. Hamam R, Hamam D, Alsaleh KA, Kassem M, Zaher W, Alfayez M, Aldahmash A and Alajez NM: Circulating microRNAs in breast cancer: Novel diagnostic and prognostic biomarkers. Cell Death Dis 8: e3045, 2017.

24. Hsiao KY, Lin YC, Gupta SK, Chang N, Yen L, Sun HS and Tsai SJ: Noncoding effects of circular RNA CCDC66 promote colon cancer growth and metastasis. Cancer Res 77: 2339-2350, 2017. 
25. Chandra Gupta S and Nandan Tripathi Y: Potential of long non-coding RNAs in cancer patients: From biomarkers to therapeutic targets. Int J Cancer 140: 1955-1967, 2017.

26. Kanwal R, Plaga AR, Liu X, Shukla GC and Gupta S: MicroRNAs in prostate cancer: Functional role as biomarkers Cancer Lett 407: 9-20, 2017.

27. Ungerleider N, Jain V, Wang Y, Maness NJ, Blair RV, Alvarez X, Midkiff C, Kolson D, Bai S, Roberts C, et al: Comparative analysis of gammaherpesvirus circRNA repertoires: Conserved and unique viral circular RNAs. J Virol 93: pii: e01952-18, 2019

28. Du WW, Zhang C, Yang W, Yong T, Awan FM and Yang BB Identifying and characterizing circRNA-protein interaction. Theranostics 7: 4183-4191, 2017.

29. Greene J, Baird AM, Brady L, Lim M, Gray SG, McDermott R and Finn SP: Circular RNAs: Biogenesis, Function and Role in Human Diseases. Front Mol Biosci 4: 38, 2017.

30. Rong D, Sun H, Li Z, Liu S, Dong C, Fu K, Tang W and Cao H: An emerging function of circRNA-miRNAs-mRNA axis in human diseases. Oncotarget 8: 73271-73281, 2017.

31. Hu J, Han Q, Gu Y, Ma J, McGrath M, Qiao F, Chen B, Song C and Ge Z: Circular RNA PVT1 expression and its roles in acute lymphoblastic leukemia. Epigenomics 10: 723-732, 2018.

32. Rupaimoole R and Slack FJ: MicroRNA therapeutics: Towards a new era for the management of cancer and other diseases. Nat Rev Drug Discov 16: 203-222, 2017.
33. Hansen TB, Kjems J and Damgaard CK: Circular RNA and miR-7 in cancer. Cancer Res 73: 5609-5612, 2013.

34. Mali SB: Review of STAT3 (Signal Transducers and Activators of Transcription) in head and neck cancer. Oral Oncol 51: 565-569, 2015.

35. Liu Y, Sepich DS and Solnica-Krezel L: Stat3/Cdc25a-dependent cell proliferation promotes embryonic axis extension during zebrafish gastrulation. PLoS Genet 13: e1006564, 2017.

36. Li J, Cui J, Zhang J, Liu Y, Han L, Jia C, Deng J and Liang H: PIAS3, an inhibitor of STAT3, has intensively negative association with the survival of gastric cancer. Int J Clin Exp Med 8: 682-689, 2015.

37. Kanda N, Seno H, Konda Y, Marusawa H, Kanai M, Nakajima T, Kawashima T, Nanakin A, Sawabu T, Uenoyama Y, et al: STAT3 is constitutively activated and supports cell survival in association with survivin expression in gastric cancer cells. Oncogene 23: 4921-4929, 2004.

38. Yu H, Lee H, Herrmann A, Buettner R and Jove R: Revisiting STAT3 signalling in cancer: New and unexpected biological functions. Nat Rev Cancer 14: 736-746, 2014.

(i) $(-)$ This work is licensed under a Creative Commons Attribution-NonCommercial-NoDerivatives 4.0 International (CC BY-NC-ND 4.0) License. 